\title{
On Impulsive Boundary Value Problems of Fractional Differential Equations with Irregular Boundary Conditions
}

\author{
Guotao Wang, ${ }^{1}$ Bashir Ahmad, ${ }^{2}$ and Lihong Zhang ${ }^{1}$ \\ ${ }^{1}$ School of Mathematics and Computer Science, Shanxi Normal University, Linfen, Shanxi 041004, China \\ ${ }^{2}$ Department of Mathematics, Faculty of Science, King Abdulaziz University, P.O. Box 80203, \\ Jeddah 21589, Saudi Arabia
}

Correspondence should be addressed to Lihong Zhang, zhanglih149@126.com

Received 25 February 2012; Accepted 4 September 2012

Academic Editor: Yong H. Wu

Copyright (C) 2012 Guotao Wang et al. This is an open access article distributed under the Creative Commons Attribution License, which permits unrestricted use, distribution, and reproduction in any medium, provided the original work is properly cited.

We study nonlinear impulsive differential equations of fractional order with irregular boundary conditions. Some existence and uniqueness results are obtained by applying standard fixed-point theorems. For illustration of the results, some examples are discussed.

\section{Introduction}

Boundary value problems of nonlinear fractional differential equations have recently been studied by several researchers. Fractional differential equations appear naturally in various fields of science and engineering and constitute an important field of research. As a matter of fact, fractional derivatives provide an excellent tool for the description of memory and hereditary properties of various materials and processes [1-4]. Some recent work on boundary value problems of fractional order can be found in [5-23] and the references therein. In [24], some existence and uniqueness results were obtained for an irregular boundary value problem of fractional differential equations.

Dynamical systems with impulse effect are regarded as a class of general hybrid systems. Impulsive hybrid systems are composed of some continuous variable dynamic systems along with certain reset maps that define impulsive switching among them. It is the switching that resets the modes and changes the continuous state of the system. There are three classes of impulsive hybrid systems, namely, impulsive differential systems [25, 26], sampled data or digital control system [27, 28], and impulsive switched system [29, 30]. 
Applications of such systems include air traffic management [31], automotive control [32,33], real-time software verification [34], transportation systems [35, 36], manufacturing [37], mobile robotics [38], and process industry [39]. In fact, hybrid systems have a central role in embedded control systems that interact with the physical world. Using hybrid models, one may represent time and event-based behaviors more accurately so as to meet challenging design requirements in the design of control systems for problems such as cut-off control and idle speed control of the engine. For more details, see [40] and the references therein.

The theory of impulsive differential equations of integer order has found its extensive applications in realistic mathematical modelling of a wide variety of practical situations and has emerged as an important area of investigation. The impulsive differential equations of fractional order have also attracted a considerable attention and a variety of results can be found in the papers [41-50].

In this paper, motivated by [24], we study a nonlinear impulsive hybrid system of fractional differential equations with irregular boundary conditions given by

$$
\begin{gathered}
{ }^{C} D^{\alpha} u(t)=f(t, u(t)), \quad 1<\alpha \leq 2, t \in J^{\prime}, \\
\Delta u\left(t_{k}\right)=I_{k}\left(u\left(t_{k}\right)\right), \quad \Delta u^{\prime}\left(t_{k}\right)=I_{k}^{*}\left(u\left(t_{k}\right)\right), \quad k=1,2, \ldots, p, \\
u^{\prime}(0)+(-1)^{\theta} u^{\prime}(T)+b u(T)=0, \quad u(0)+(-1)^{\theta+1} u(T)=0, \quad \theta=1,2,
\end{gathered}
$$

where ${ }^{C} D^{\alpha}$ is the Caputo fractional derivative, $f \in C(J \times \mathbb{R}, \mathbb{R}), I_{k}, I_{k}^{*} \in C(\mathbb{R}, \mathbb{R}), b \in$ $\mathbb{R}, b \neq 0, J=[0, T](T>0), 0=t_{0}<t_{1}<\cdots<t_{k}<\cdots<t_{p}<t_{p+1}=T, J^{\prime}=$ $J \backslash\left\{t_{1}, t_{2}, \ldots, t_{p}\right\}, \Delta u\left(t_{k}\right)=u\left(t_{k}^{+}\right)-u\left(t_{k}^{-}\right)$, where $u\left(t_{k}^{+}\right)$and $u\left(t_{k}^{-}\right)$denote the right and the left limits of $u(t)$ at $t=t_{k}(k=1,2, \ldots, p)$, respectively. $\Delta u^{\prime}\left(t_{k}\right)$ have a similar meaning for $u^{\prime}(t)$.

Here, we remark that irregular boundary value problems for ordinary and partial differential equations occur in scientific and engineering disciplines and have been addressed by many authors, for instance, see [24] and the references.

The paper is organized as follows. Section 2 deals with some definitions and preliminary results, while the main results are presented in Section 3.

\section{Preliminaries}

Let us fix $J_{0}=\left[0, t_{1}\right], J_{k-1}=\left(t_{k-1}, t_{k}\right], k=2, \ldots, p+1$ with $t_{p+1}=T$ and introduce the spaces:

$$
P C(J, \mathbb{R})=\left\{u: J \longrightarrow \mathbb{R} \mid u \in C\left(J_{k}\right), k=0,1, \ldots, p \text {, and } u\left(t_{k}^{+}\right) \text {exist }, k=1,2, \ldots, p\right\}
$$

with the norm $\|u\|=\sup _{t \in J}|u(t)|$, and

$$
P C^{1}(J, \mathbb{R})=\left\{\begin{array}{c}
u: J \rightarrow \mathbb{R} \mid u \in C^{1}\left(J_{k}\right), k=0,1, \ldots, p, \\
\text { and } u\left(t_{k}^{+}\right), u^{\prime}\left(t_{k}^{+}\right) \text {exist }, k=1,2, \ldots, p
\end{array}\right\}
$$

with the norm $\|u\|_{P C^{1}}=\max \left\{\|u\|,\left\|u^{\prime}\right\|\right\}$. Obviously, $P C(J, \mathbb{R})$ and $P C^{1}(J, \mathbb{R})$ are Banach spaces.

Definition 2.1. A function $u \in P C^{1}(J, \mathbb{R})$ with its Caputo derivative of order $\alpha$ existing on $J$ is a solution of (1.1) if it satisfies (1.1). 
To prove the existence of solutions of problem (1.1), we need the following fixed-point theorems.

Theorem 2.2 (see [51]). Let $E$ be a Banach space. Assume that $\Omega$ is an open bounded subset of $E$ with $\theta \in \Omega$ and let $T: \bar{\Omega} \rightarrow$ E be a completely continuous operator such that

$$
\|T u\| \leq\|u\|, \quad \forall u \in \partial \Omega .
$$

Then $T$ has a fixed point in $\bar{\Omega}$.

Lemma 2.3 (see [1]). For $\alpha>0$, the general solution of fractional differential equation ${ }^{C} D^{\alpha} u(t)=0$ is

$$
u(t)=C_{0}+C_{1} t+C_{2} t^{2}+\cdots+C_{n-1} t^{n-1},
$$

where $C_{i} \in \mathbb{R}, i=0,1,2, \ldots, n-1, n=[\alpha]+1([\alpha]$ denotes integer part of $\alpha)$.

Lemma 2.4 (see [1]). Let $\alpha>0$. Then

$$
I^{\alpha}{ }^{C} D^{\alpha} u(t)=u(t)+C_{0}+C_{1} t+C_{2} t^{2}+\cdots+C_{n-1} t^{n-1}
$$

for some $C_{i} \in \mathbb{R}, i=1,2, \ldots, n-1, n=[\alpha]+1$.

Lemma 2.5. For a given $y \in C[0, T]$, a function $u$ is a solution of the following impulsive irregular boundary value problem

$$
\begin{gathered}
{ }^{C} D^{\alpha} u(t)=y(t), \quad 1<\alpha \leq 2, t \in J^{\prime}, \\
\Delta u\left(t_{k}\right)=I_{k}\left(u\left(t_{k}\right)\right), \quad \Delta u^{\prime}\left(t_{k}\right)=I_{k}^{*}\left(u\left(t_{k}\right)\right), \quad k=1,2, \ldots, p, \\
u^{\prime}(0)+(-1)^{\theta} u^{\prime}(T)+b u(T)=0, \quad u(0)+(-1)^{\theta+1} u(T)=0, \quad \theta=1,2, \quad b \neq 0,
\end{gathered}
$$


if and only if $u$ is a solution of the impulsive fractional integral equation

$$
u(t)=\left\{\begin{array}{l}
\int_{0}^{t} \frac{(t-s)^{\alpha-1}}{\Gamma(\alpha)} y(s) d s+\frac{1-(-1)^{\theta+1}}{b T} \int_{t_{p}}^{T} \frac{(T-s)^{\alpha-1}}{\Gamma(\alpha)} y(s) d s \\
\quad+\frac{\left[1+(-1)^{\theta+1}\right] t}{b T} \int_{t_{p}}^{T} \frac{(T-s)^{\alpha-2}}{\Gamma(\alpha-1)} y(s) d s \\
\quad-\frac{1}{b} \int_{t_{p}}^{T} \frac{(T-s)^{\alpha-2}}{\Gamma(\alpha-1)} y(s) d s-\frac{t}{T} \int_{t_{p}}^{T} \frac{(T-s)^{\alpha-1}}{\Gamma(\alpha)} y(s) d s+\mathcal{A}, \quad t \in J_{0} ; \\
\int_{t_{k}}^{t} \frac{(t-s)^{\alpha-1}}{\Gamma(\alpha)} y(s) d s+\frac{1-(-1)^{\theta+1}}{b T} \int_{t_{p}}^{T} \frac{(T-s)^{\alpha-1}}{\Gamma(\alpha)} y(s) d s \\
+\frac{\left[1+(-1)^{\theta+1}\right] t}{b T} \int_{t_{p}}^{T} \frac{(T-s)^{\alpha-2}}{\Gamma(\alpha-1)} y(s) d s \\
\quad-\frac{1}{b} \int_{t_{p}}^{T} \frac{(T-s)^{\alpha-2}}{\Gamma(\alpha-1)} y(s) d s-\frac{t}{T} \int_{t_{p}}^{T} \frac{(T-s)^{\alpha-1}}{\Gamma(\alpha)} y(s) d s \\
\quad+\sum_{i=1}^{k}\left[\int_{t_{i-1}}^{t_{i}} \frac{\left(t_{i}-s\right)^{\alpha-1}}{\Gamma(\alpha)} y(s) d s+I_{i}\left(u\left(t_{i}\right)\right)\right] \\
\quad+\sum_{i=1}^{k-1}\left(t_{k}-t_{i}\right)\left[\int_{t_{i-1}}^{t_{i}} \frac{\left(t_{i}-s\right)^{\alpha-2}}{\Gamma(\alpha-1)} y(s) d s+I_{i}^{*}\left(u\left(t_{i}\right)\right)\right] \\
\quad+\sum_{i=1}^{k}\left(t-t_{k}\right)\left[\int_{t_{i-1}}^{t_{i}} \frac{\left(t_{i}-s\right)^{\alpha-2}}{\Gamma(\alpha-1)} y(s) d s+I_{i}^{*}\left(u\left(t_{i}\right)\right)\right]+\mathcal{A}, \\
\end{array}\right.
$$

where

$$
\begin{aligned}
\mathcal{A}=\frac{\left[1+(-1)^{\theta+1}\right] t-T}{b T} \sum_{i=1}^{p} & {\left[\int_{t_{i-1}}^{t_{i}} \frac{\left(t_{i}-s\right)^{\alpha-2}}{\Gamma(\alpha-1)} y(s) d s+I_{i}^{*}\left(u\left(t_{i}\right)\right)\right] } \\
+\frac{1-(-1)^{\theta+1}-b t}{b T}\left\{\sum_{i=1}^{p}\right. & {\left[\int_{t_{i-1}}^{t_{i}} \frac{\left(t_{i}-s\right)^{\alpha-1}}{\Gamma(\alpha)} y(s) d s+I_{i}\left(u\left(t_{i}\right)\right)\right] } \\
& +\sum_{i=1}^{p-1}\left(t_{p}-t_{i}\right)\left[\int_{t_{i-1}}^{t_{i}} \frac{\left(t_{i}-s\right)^{\alpha-2}}{\Gamma(\alpha-1)} y(s) d s+I_{i}^{*}\left(u\left(t_{i}\right)\right)\right] \\
& \left.+\sum_{i=1}^{p}\left(T-t_{p}\right)\left[\int_{t_{i-1}}^{t_{i}} \frac{\left(t_{i}-s\right)^{\alpha-2}}{\Gamma(\alpha-1)} y(s) d s+I_{i}^{*}\left(u\left(t_{i}\right)\right)\right]\right\} .
\end{aligned}
$$

Proof. Let $u$ be a solution of (2.6). Then, by Lemma 2.4, we have

$$
u(t)=I^{\alpha} y(t)-c_{1}-c_{2} t=\frac{1}{\Gamma(\alpha)} \int_{0}^{t}(t-s)^{\alpha-1} y(s) d s-c_{1}-c_{2} t, \quad t \in J_{0}
$$


for some $c_{1}, c_{2} \in \mathbb{R}$. Differentiating (2.9), we get

$$
u^{\prime}(t)=\frac{1}{\Gamma(\alpha-1)} \int_{0}^{t}(t-s)^{\alpha-2} y(s) d s-c_{2}, \quad t \in J_{0} .
$$

If $t \in J_{1}$, then

$$
\begin{gathered}
u(t)=\frac{1}{\Gamma(\alpha)} \int_{t_{1}}^{t}(t-s)^{\alpha-1} y(s) d s-d_{1}-d_{2}\left(t-t_{1}\right), \\
u^{\prime}(t)=\frac{1}{\Gamma(\alpha-1)} \int_{t_{1}}^{t}(t-s)^{\alpha-2} y(s) d s-d_{2},
\end{gathered}
$$

for some $d_{1}, d_{2} \in \mathbb{R}$. Thus,

$$
\begin{array}{ll}
u\left(t_{1}^{-}\right)=\frac{1}{\Gamma(\alpha)} \int_{0}^{t_{1}}\left(t_{1}-s\right)^{\alpha-1} y(s) d s-c_{1}-c_{2} t_{1}, & u\left(t_{1}^{+}\right)=-d_{1} \\
u^{\prime}\left(t_{1}^{-}\right)=\frac{1}{\Gamma(\alpha-1)} \int_{0}^{t_{1}}\left(t_{1}-s\right)^{\alpha-2} y(s) d s-c_{2}, & u^{\prime}\left(t_{1}^{+}\right)=-d_{2} .
\end{array}
$$

Using the impulse conditions

$$
\Delta u\left(t_{1}\right)=u\left(t_{1}^{+}\right)-u\left(t_{1}^{-}\right)=I_{1}\left(u\left(t_{1}\right)\right), \quad \Delta u^{\prime}\left(t_{1}\right)=u^{\prime}\left(t_{1}^{+}\right)-u^{\prime}\left(t_{1}^{-}\right)=I_{1}^{*}\left(u\left(t_{1}\right)\right),
$$

we find that

$$
\begin{aligned}
& -d_{1}=\frac{1}{\Gamma(\alpha)} \int_{0}^{t_{1}}\left(t_{1}-s\right)^{\alpha-1} y(s) d s-c_{1}-c_{2} t_{1}+I_{1}\left(u\left(t_{1}\right)\right) \\
& -d_{2}=\frac{1}{\Gamma(\alpha-1)} \int_{0}^{t_{1}}\left(t_{1}-s\right)^{\alpha-2} y(s) d s-c_{2}+I_{1}^{*}\left(u\left(t_{1}\right)\right)
\end{aligned}
$$

Consequently, we obtain

$$
\begin{aligned}
u(t)= & \frac{1}{\Gamma(\alpha)} \int_{t_{1}}^{t}(t-s)^{\alpha-1} y(s) d s+\frac{1}{\Gamma(\alpha)} \int_{0}^{t_{1}}\left(t_{1}-s\right)^{\alpha-1} y(s) d s \\
& +\frac{t-t_{1}}{\Gamma(\alpha-1)} \int_{0}^{t_{1}}\left(t_{1}-s\right)^{\alpha-2} y(s) d s+I_{1}\left(u\left(t_{1}\right)\right)+\left(t-t_{1}\right) I_{1}^{*}\left(u\left(t_{1}\right)\right)-c_{1}-c_{2} t, \quad t \in J_{1} .
\end{aligned}
$$


By a similar process, we get

$$
\begin{aligned}
u(t)= & \int_{t_{k}}^{t} \frac{(t-s)^{\alpha-1}}{\Gamma(\alpha)} y(s) d s+\sum_{i=1}^{k}\left[\int_{t_{i-1}}^{t_{i}} \frac{\left(t_{i}-s\right)^{\alpha-1}}{\Gamma(\alpha)} y(s) d s+I_{i}\left(u\left(t_{i}\right)\right)\right] \\
& +\sum_{i=1}^{k-1}\left(t_{k}-t_{i}\right)\left[\int_{t_{i-1}}^{t_{i}} \frac{\left(t_{i}-s\right)^{\alpha-2}}{\Gamma(\alpha-1)} y(s) d s+I_{i}^{*}\left(u\left(t_{i}\right)\right)\right] \\
& +\sum_{i=1}^{k}\left(t-t_{k}\right)\left[\int_{t_{i-1}}^{t_{i}} \frac{\left(t_{i}-s\right)^{\alpha-2}}{\Gamma(\alpha-1)} y(s) d s+I_{i}^{*}\left(u\left(t_{i}\right)\right)\right]-c_{1}-c_{2} t, \quad t \in J_{k}, k=1,2, \ldots, p
\end{aligned}
$$

Applying the boundary conditions $u^{\prime}(0)+(-1)^{\theta} u^{\prime}(T)+b u(T)=0$ and $u(0)+$ $(-1)^{\theta+1} u(T)=0$, we find that

$$
\begin{aligned}
& c_{1}=-\frac{1-(-1)^{\theta+1}}{b T} \int_{t_{p}}^{T} \frac{(T-s)^{\alpha-1}}{\Gamma(\alpha)} y(s) d s+\frac{1}{b} \int_{t_{p}}^{T} \frac{(T-s)^{\alpha-2}}{\Gamma(\alpha-1)} y(s) d s \\
& +\sum_{i=1}^{p} \frac{1}{b}\left[\int_{t_{i-1}}^{t_{i}} \frac{\left(t_{i}-s\right)^{\alpha-2}}{\Gamma(\alpha-1)} y(s) d s+I_{i}^{*}\left(u\left(t_{i}\right)\right)\right] \\
& -\frac{1-(-1)^{\theta+1}}{b T}\left\{\sum_{i=1}^{p}\left[\int_{t_{i-1}}^{t_{i}} \frac{\left(t_{i}-s\right)^{\alpha-1}}{\Gamma(\alpha)} y(s) d s+I_{i}\left(u\left(t_{i}\right)\right)\right]\right. \\
& +\sum_{i=1}^{p-1}\left(t_{p}-t_{i}\right)\left[\int_{t_{i-1}}^{t_{i}} \frac{\left(t_{i}-s\right)^{\alpha-2}}{\Gamma(\alpha-1)} y(s) d s+I_{i}^{*}\left(u\left(t_{i}\right)\right)\right] \\
& \left.+\sum_{i=1}^{p}\left(T-t_{p}\right)\left[\int_{t_{i-1}}^{t_{i}} \frac{\left(t_{i}-s\right)^{\alpha-2}}{\Gamma(\alpha-1)} y(s) d s+I_{i}^{*}\left(u\left(t_{i}\right)\right)\right]\right\}, \\
& c_{2}=-\frac{1+(-1)^{\theta+1}}{b T} \int_{t_{p}}^{T} \frac{(T-s)^{\alpha-2}}{\Gamma(\alpha-1)} y(s) d s+\frac{1}{T} \int_{t_{p}}^{T} \frac{(T-s)^{\alpha-1}}{\Gamma(\alpha)} y(s) d s \\
& -\frac{1+(-1)^{\theta+1}}{b T} \sum_{i=1}^{p}\left[\int_{t_{i-1}}^{t_{i}} \frac{\left(t_{i}-s\right)^{\alpha-2}}{\Gamma(\alpha-1)} y(s) d s+I_{i}^{*}\left(u\left(t_{i}\right)\right)\right] \\
& +\frac{1}{T}\left\{\sum_{i=1}^{p}\left[\int_{t_{i-1}}^{t_{i}} \frac{\left(t_{i}-s\right)^{\alpha-1}}{\Gamma(\alpha)} y(s) d s+I_{i}\left(u\left(t_{i}\right)\right)\right]\right. \\
& +\sum_{i=1}^{p-1}\left(t_{p}-t_{i}\right)\left[\int_{t_{i-1}}^{t_{i}} \frac{\left(t_{i}-s\right)^{\alpha-2}}{\Gamma(\alpha-1)} y(s) d s+I_{i}^{*}\left(u\left(t_{i}\right)\right)\right] \\
& \left.+\sum_{i=1}^{p}\left(T-t_{p}\right)\left[\int_{t_{i-1}}^{t_{i}} \frac{\left(t_{i}-s\right)^{\alpha-2}}{\Gamma(\alpha-1)} y(s) d s+I_{i}^{*}\left(u\left(t_{i}\right)\right)\right]\right\} .
\end{aligned}
$$


Substituting the value of $c_{i}(i=1,2)$ in (2.9) and (2.16), we obtain (2.7). Conversely, assume that $u$ is a solution of the impulsive fractional integral equation (2.7), then by a direct computation, it follows that the solution given by (2.7) satisfies (2.6). This completes the proof.

Remark 2.6. With $T=\pi$, the first five terms of the solution (2.7) correspond to the solution for the problem without impulses [24].

\section{Main Results}

Define an operator $\mathcal{G}: P C(J, \mathbb{R}) \rightarrow P C(J, \mathbb{R})$ by

$$
\begin{aligned}
\mathcal{G} u(t)= & \int_{t_{k}}^{t} \frac{(t-s)^{\alpha-1}}{\Gamma(\alpha)} f(s, u(s)) d s+\sum_{i=1}^{k}\left[\int_{t_{i-1}}^{t_{i}} \frac{\left(t_{i}-s\right)^{\alpha-1}}{\Gamma(\alpha)} f(s, u(s)) d s+I_{i}\left(u\left(t_{i}\right)\right)\right] \\
& +\sum_{i=1}^{k-1}\left(t_{k}-t_{i}\right)\left[\int_{t_{i-1}}^{t_{i}} \frac{\left(t_{i}-s\right)^{\alpha-2}}{\Gamma(\alpha-1)} f(s, u(s)) d s+I_{i}^{*}\left(u\left(t_{i}\right)\right)\right] \\
& +\sum_{i=1}^{k}\left(t-t_{k}\right)\left[\int_{t_{i-1}}^{t_{i}} \frac{\left(t_{i}-s\right)^{\alpha-2}}{\Gamma(\alpha-1)} f(s, u(s)) d s+I_{i}^{*}\left(u\left(t_{i}\right)\right)\right] \\
& +\frac{1-(-1)^{\theta+1}}{b T} \int_{t_{p}}^{T} \frac{(T-s)^{\alpha-1}}{\Gamma(\alpha)} f(s, u(s)) d s+\frac{\left[1+(-1)^{\theta+1}\right] t}{b T} \int_{t_{p}}^{T} \frac{(T-s)^{\alpha-2}}{\Gamma(\alpha-1)} f(s, u(s)) d s \\
& -\frac{1}{b} \int_{t_{p}}^{T} \frac{(T-s)^{\alpha-2}}{\Gamma(\alpha-1)} f(s, u(s)) d s-\frac{t}{T} \int_{t_{p}}^{T} \frac{(T-s)^{\alpha-1}}{\Gamma(\alpha)} f(s, u(s)) d s \\
& +\frac{\left[1+(-1)^{\theta+1}\right] t-T}{b T} \sum_{i=1}^{p}\left[\int_{t_{i-1}}^{t_{i}} \frac{\left(t_{i}-s\right)^{\alpha-2}}{\Gamma(\alpha-1)} f(s, u(s)) d s+I_{i}^{*}\left(u\left(t_{i}\right)\right)\right] \\
& +\frac{1-(-1)^{\theta+1}-b t}{b T}\left\{\sum_{i=1}^{p}\left[\int_{t_{i-1}}^{t_{i}} \frac{\left(t_{i}-s\right)^{\alpha-1}}{\Gamma(\alpha)} f(s, u(s)) d s+I_{i}\left(u\left(t_{i}\right)\right)\right]\right. \\
& +\sum_{i=1}^{p-1}\left(t_{p}-t_{i}\right)\left[\int_{t_{i-1}}^{t_{i}} \frac{\left(t_{i}-s\right)^{\alpha-2}}{\Gamma(\alpha-1)} f(s, u(s)) d s+I_{i}^{*}\left(u\left(t_{i}\right)\right)\right] \\
& \\
& \\
& \\
&
\end{aligned}
$$

Notice that problem (1.1) has a solution if and only if the operator $\mathcal{G}$ has a fixed point. 
For the sake of convenience, we set the following notations:

$$
\begin{gathered}
\mu=2(1+p)\left(1+\frac{1}{|b| T}\right) I^{\alpha} a(T)+\left[2(2 p-1) T+\frac{5 p+1}{|b|}\right] I^{\alpha-1} \\
+2\left(1+\frac{1}{|b| T}\right) p L_{2}+\left[2(2 p-1) T+\frac{5 p-2}{|b|}\right] L_{3} \\
v=2(1+p)\left(1+\frac{1}{|b| T}\right) \frac{T^{\alpha}}{\Gamma(\alpha+1)}+\left[2(2 p-1) T+\frac{5 p+1}{|b|}\right] \frac{T^{\alpha-1}}{\Gamma(\alpha)}
\end{gathered}
$$

Theorem 3.1. Assume that

$\left(\mathbf{H}_{1}\right)$ there exists a nonnegative function $a(t) \in L(0, T)$ such that

$$
|f(t, u)| \leq a(t)+\xi|u|^{\rho}, \quad 0<\rho<1,
$$

where $\xi$ is a nonnegative constant;

$\left(\mathbf{H}_{2}\right)$ there exist positive constants $L_{2}$ and $L_{3}$ such that

$$
\left|I_{k}(u)\right| \leq L_{2}, \quad\left|I_{k}^{*}(u)\right| \leq L_{3}, \quad \text { for } t \in J, u \in \mathbb{R}, k=1,2, \ldots, p \text {. }
$$

Then problem (1.1) has at least one solution.

Proof. As a first step, we show that the operator $\mathcal{G}: P C(J, \mathbb{R}) \rightarrow P C(J, \mathbb{R})$ is completely continuous. Observe that continuity of $\mathcal{G}$ follows from the continuity of $f, I_{k}$ and $I_{k}^{*}$.

Let $\Omega \subset P C(J, \mathbb{R})$ be bounded. Then, there exist positive constants $L_{i}>0(i=1,2,3)$ such that $|f(t, u)| \leq L_{1},\left|I_{k}(u)\right| \leq L_{2}$, and $\left|I_{k}^{*}(u)\right| \leq L_{3}$, for all $u \in \Omega$. Thus, for all $u \in \Omega$, we have

$$
\begin{aligned}
|\mathcal{G} u(t)| \leq & \int_{t_{k}}^{t} \frac{(t-s)^{\alpha-1}}{\Gamma(\alpha)}|f(s, u(s))| d s+\sum_{i=1}^{k}\left[\int_{t_{i-1}}^{t_{i}} \frac{\left(t_{i}-s\right)^{\alpha-1}}{\Gamma(\alpha)}|f(s, u(s))| d s+\left|I_{i}\left(u\left(t_{i}\right)\right)\right|\right] \\
& +\sum_{i=1}^{k-1}\left(t_{k}-t_{i}\right)\left[\int_{t_{i-1}}^{t_{i}} \frac{\left(t_{i}-s\right)^{\alpha-2}}{\Gamma(\alpha-1)}|f(s, u(s))| d s+\left|I_{i}^{*}\left(u\left(t_{i}\right)\right)\right|\right] \\
& +\sum_{i=1}^{k}\left(t-t_{k}\right)\left[\int_{t_{i-1}}^{t_{i}} \frac{\left(t_{i}-s\right)^{\alpha-2}}{\Gamma(\alpha-1)}|f(s, u(s))| d s+\left|I_{i}^{*}\left(u\left(t_{i}\right)\right)\right|\right]
\end{aligned}
$$


Abstract and Applied Analysis

$$
\begin{aligned}
& +\frac{1-(-1)^{\theta+1}}{|b| T} \int_{t_{p}}^{T} \frac{(T-s)^{\alpha-1}}{\Gamma(\alpha)}|f(s, u(s))| d s \\
& +\frac{\left[1+(-1)^{\theta+1}\right] t}{|b| T} \int_{t_{p}}^{T} \frac{(T-s)^{\alpha-2}}{\Gamma(\alpha-1)}|f(s, u(s))| d s \\
& +\frac{1}{|b|} \int_{t_{p}}^{T} \frac{(T-s)^{\alpha-2}}{\Gamma(\alpha-1)}|f(s, u(s))| d s+\frac{t}{T} \int_{t_{p}}^{T} \frac{(T-s)^{\alpha-1}}{\Gamma(\alpha)}|f(s, u(s))| d s \\
& +\left|\frac{\left[1+(-1)^{\theta+1}\right] t-T}{b T}\right| \sum_{i=1}^{p}\left[\int_{t_{i-1}}^{t_{i}} \frac{\left(t_{i}-s\right)^{\alpha-2}}{\Gamma(\alpha-1)}|f(s, u(s))| d s+\left|I_{i}^{*}\left(u\left(t_{i}\right)\right)\right|\right] \\
& +\left|\frac{1-(-1)^{\theta+1}-b t}{b T}\right|\left\{\sum_{i=1}^{p}\left[\int_{t_{i-1}}^{t_{i}} \frac{\left(t_{i}-s\right)^{\alpha-1}}{\Gamma(\alpha)}|f(s, u(s))| d s+\left|I_{i}\left(u\left(t_{i}\right)\right)\right|\right]\right. \\
& +\sum_{i=1}^{p-1}\left(t_{p}-t_{i}\right)\left[\int_{t_{i-1}}^{t_{i}} \frac{\left(t_{i}-s\right)^{\alpha-2}}{\Gamma(\alpha-1)}|f(s, u(s))| d s+\left|I_{i}^{*}\left(u\left(t_{i}\right)\right)\right|\right] \\
& \left.+\sum_{i=1}^{p}\left(T-t_{p}\right)\left[\int_{t_{i-1}}^{t_{i}} \frac{\left(t_{i}-s\right)^{\alpha-2}}{\Gamma(\alpha-1)}|f(s, u(s))| d s+\left|I_{i}^{*}\left(u\left(t_{i}\right)\right)\right|\right]\right\} \\
& \leq L_{1} \int_{t_{k}}^{t} \frac{(t-s)^{\alpha-1}}{\Gamma(\alpha)} d s+\sum_{i=1}^{p}\left[L_{1} \int_{t_{i-1}}^{t_{i}} \frac{\left(t_{i}-s\right)^{\alpha-1}}{\Gamma(\alpha)} d s+L_{2}\right] \\
& +\sum_{i=1}^{p-1} T\left[L_{1} \int_{t_{i-1}}^{t_{i}} \frac{\left(t_{i}-s\right)^{\alpha-2}}{\Gamma(\alpha-1)} d s+L_{3}\right] \\
& +\sum_{i=1}^{p} T\left[L_{1} \int_{t_{i-1}}^{t_{i}} \frac{\left(t_{i}-s\right)^{\alpha-2}}{\Gamma(\alpha-1)} d s+L_{3}\right]+\frac{2 L_{1}}{|b| T} \int_{t_{p}}^{T} \frac{(T-s)^{\alpha-1}}{\Gamma(\alpha)} d s+\frac{2 L_{1}}{|b|} \int_{t_{p}}^{T} \frac{(T-s)^{\alpha-2}}{\Gamma(\alpha-1)} d s \\
& +\frac{L_{1}}{|b|} \int_{t_{p}}^{T} \frac{(T-s)^{\alpha-2}}{\Gamma(\alpha-1)} d s+L_{1} \int_{t_{p}}^{T} \frac{(T-s)^{\alpha-1}}{\Gamma(\alpha)} d s+\frac{1}{|b|} \sum_{i=1}^{p}\left[L_{1} \int_{t_{i-1}}^{t_{i}} \frac{\left(t_{i}-s\right)^{\alpha-2}}{\Gamma(\alpha-1)} d s+L_{3}\right] \\
& +\frac{2+|b| T}{|b| T}\left\{\sum_{i=1}^{p}\left[L_{1} \int_{t_{i-1}}^{t_{i}} \frac{\left(t_{i}-s\right)^{\alpha-1}}{\Gamma(\alpha)} d s+L_{2}\right]+\sum_{i=1}^{p-1} T\left[L_{1} \int_{t_{i-1}}^{t_{i}} \frac{\left(t_{i}-s\right)^{\alpha-2}}{\Gamma(\alpha-1)} d s+L_{3}\right]\right. \\
& \left.+\sum_{i=1}^{p} T\left[L_{1} \int_{t_{i-1}}^{t_{i}} \frac{\left(t_{i}-s\right)^{\alpha-2}}{\Gamma(\alpha-1)} d s+L_{3}\right]\right\} \\
& \leq \frac{1}{|b|}\left\{\frac{2(1+p)(|b| T+1) T^{\alpha-1} L_{1}}{\Gamma(\alpha+1)}+\frac{[5 p+1+2|b| T(2 p-1)] T^{\alpha-1} L_{1}}{\Gamma(\alpha)}\right. \\
& \left.+\frac{2(1+|b| T) p L_{2}}{T}+[5 p-2+2|b| T(2 p-1)] L_{3}\right\},
\end{aligned}
$$


which implies

$$
\begin{aligned}
\| \mathcal{G}_{u} u \leq \frac{1}{|b|}\{ & \frac{2(1+p)(|b| T+1) T^{\alpha-1} L_{1}}{\Gamma(\alpha+1)}+\frac{[5 p+1+2|b| T(2 p-1)] T^{\alpha-1} L_{1}}{\Gamma(\alpha)}+\frac{2(1+|b| T) p L_{2}}{T} \\
& \left.+[5 p-2+2|b| T(2 p-1)] L_{3}\right\}:=L .
\end{aligned}
$$

On the other hand, for any $t \in J_{k}, 0 \leq k \leq p$, we get

$$
\begin{aligned}
& \left|(\mathcal{G} u)^{\prime}(t)\right| \leq \int_{t_{k}}^{t} \frac{(t-s)^{\alpha-2}}{\Gamma(\alpha-1)}|f(s, u(s))| d s+\sum_{i=1}^{k}\left[\int_{t_{i-1}}^{t_{i}} \frac{\left(t_{i}-s\right)^{\alpha-2}}{\Gamma(\alpha-1)}|f(s, u(s))| d s+\left|I_{i}^{*}\left(u\left(t_{i}\right)\right)\right|\right] \\
& +\frac{\left[1+(-1)^{\theta+1}\right]}{|b| T} \int_{t_{p}}^{T} \frac{(T-s)^{\alpha-2}}{\Gamma(\alpha-1)}|f(s, u(s))| d s+\frac{1}{T} \int_{t_{p}}^{T} \frac{(T-s)^{\alpha-1}}{\Gamma(\alpha)}|f(s, u(s))| d s \\
& +\frac{\left[1+(-1)^{\theta+1}\right]}{|b| T} \sum_{i=1}^{p}\left[\int_{t_{i-1}}^{t_{i}} \frac{\left(t_{i}-s\right)^{\alpha-2}}{\Gamma(\alpha-1)}|f(s, u(s))| d s+\left|I_{i}^{*}\left(u\left(t_{i}\right)\right)\right|\right] \\
& +\frac{1}{T}\left\{\sum_{i=1}^{p}\left[\int_{t_{i-1}}^{t_{i}} \frac{\left(t_{i}-s\right)^{\alpha-1}}{\Gamma(\alpha)}|f(s, u(s))| d s+\left|I_{i}\left(u\left(t_{i}\right)\right)\right|\right]\right. \\
& +\sum_{i=1}^{p-1}\left(t_{p}-t_{i}\right)\left[\int_{t_{i-1}}^{t_{i}} \frac{\left(t_{i}-s\right)^{\alpha-2}}{\Gamma(\alpha-1)}|f(s, u(s))| d s+\left|I_{i}^{*}\left(u\left(t_{i}\right)\right)\right|\right] \\
& \left.+\sum_{i=1}^{p}\left(T-t_{p}\right)\left[\int_{t_{i-1}}^{t_{i}} \frac{\left(t_{i}-s\right)^{\alpha-2}}{\Gamma(\alpha-1)}|f(s, u(s))| d s+\left|I_{i}^{*}\left(u\left(t_{i}\right)\right)\right|\right]\right\} \\
& \leq L_{1} \int_{t_{k}}^{t} \frac{(t-s)^{\alpha-2}}{\Gamma(\alpha-1)} d s+\sum_{i=1}^{p}\left[L_{1} \int_{t_{i-1}}^{t_{i}} \frac{\left(t_{i}-s\right)^{\alpha-2}}{\Gamma(\alpha-1)} d s+L_{3}\right]+\frac{2 L_{1}}{|b| T} \int_{t_{p}}^{T} \frac{(T-s)^{\alpha-2}}{\Gamma(\alpha-1)} d s \\
& +\frac{L_{1}}{T} \int_{t_{p}}^{T} \frac{(T-s)^{\alpha-1}}{\Gamma(\alpha)} d s+\frac{2}{|b| T} \sum_{i=1}^{p}\left[L_{1} \int_{t_{i-1}}^{t_{i}} \frac{\left(t_{i}-s\right)^{\alpha-2}}{\Gamma(\alpha-1)} d s+L_{3}\right] \\
& +\frac{1}{T}\left\{\sum_{i=1}^{p}\left[L_{1} \int_{t_{i-1}}^{t_{i}} \frac{\left(t_{i}-s\right)^{\alpha-1}}{\Gamma(\alpha)} d s+L_{2}\right]+\sum_{i=1}^{p-1} T\left[L_{1} \int_{t_{i-1}}^{t_{i}} \frac{\left(t_{i}-s\right)^{\alpha-2}}{\Gamma(\alpha-1)} d s+L_{3}\right]\right. \\
& \left.+\sum_{i=1}^{p} T\left[L_{1} \int_{t_{i-1}}^{t_{i}} \frac{\left(t_{i}-s\right)^{\alpha-2}}{\Gamma(\alpha-1)} d s+L_{3}\right]\right\} \\
& \leq \frac{(1+p) T^{\alpha-1} L_{1}}{\Gamma(\alpha+1)}+\frac{3 p T^{\alpha-1} L_{1}}{\Gamma(\alpha)}+\frac{2(1+2 p) T^{\alpha-2} L_{1}}{|b| \Gamma(\alpha)}+\frac{p L_{2}}{T}+\frac{2 p L_{3}}{|b| T}+(3 p-1) L_{3}:=\bar{L} .
\end{aligned}
$$


Hence, for $t_{1}, t_{2} \in J_{k}$ with $t_{1}<t_{2}, 0 \leq k \leq p$, we have

$$
\left|(\mathcal{G} u)\left(t_{2}\right)-(\mathcal{G} u)\left(t_{1}\right)\right| \leq \int_{t_{1}}^{t_{2}}\left|(\mathcal{G} u)^{\prime}(s)\right| d s \leq \bar{L}\left(t_{2}-t_{1}\right)
$$

This implies that $G$ is equicontinuous on all $J_{k}, k=0,1,2, \ldots, p$ and hence, by the ArzelaAscoli theorem, the operator $\mathcal{G}: P C(J, \mathbb{R}) \rightarrow P C(J, \mathbb{R})$ is completely continuous.

Next, we prove that $\mathcal{G}: \mathbb{B} \rightarrow \mathbb{B}$. For that, let us choose $R \geq \max \left\{2 \mu,(2 v \xi)^{1 /(1-\rho)}\right\}$ and define a ball $\mathcal{B}=\{u \in P C(J, \mathbb{R}):\|u\| \leq R\}$. For any $u \in \mathcal{B}$, by the assumptions $\left(\mathbf{H}_{1}\right)$ and $\left(\mathbf{H}_{2}\right)$, we have

$$
\begin{aligned}
& |\mathcal{G} u(t)| \leq \int_{t_{k}}^{t} \frac{(t-s)^{\alpha-1}}{\Gamma(\alpha)}\left[a(s)+\xi|u(s)|^{\rho}\right] d s+\sum_{i=1}^{k}\left[\int_{t_{i-1}}^{t_{i}} \frac{\left(t_{i}-s\right)^{\alpha-1}}{\Gamma(\alpha)}\left[a(s)+\xi|u(s)|^{\rho}\right] d s+\left|I_{i}\left(u\left(t_{i}\right)\right)\right|\right] \\
& +\sum_{i=1}^{k-1}\left(t_{k}-t_{i}\right)\left[\int_{t_{i-1}}^{t_{i}} \frac{\left(t_{i}-s\right)^{\alpha-2}}{\Gamma(\alpha-1)}\left[a(s)+\xi|u(s)|^{\rho}\right] d s+\left|I_{i}^{*}\left(u\left(t_{i}\right)\right)\right|\right] \\
& +\sum_{i=1}^{k}\left(t-t_{k}\right)\left[\int_{t_{i-1}}^{t_{i}} \frac{\left(t_{i}-s\right)^{\alpha-2}}{\Gamma(\alpha-1)}\left[a(s)+\xi|u(s)|^{\rho}\right] d s+\left|I_{i}^{*}\left(u\left(t_{i}\right)\right)\right|\right] \\
& +\frac{1-(-1)^{\theta+1}}{|b| T} \int_{t_{p}}^{T} \frac{(T-s)^{\alpha-1}}{\Gamma(\alpha)}\left[a(s)+\xi|u(s)|^{\rho}\right] d s \\
& +\frac{\left[1+(-1)^{\theta+1}\right] t}{|b| T} \int_{t_{p}}^{T} \frac{(T-s)^{\alpha-2}}{\Gamma(\alpha-1)}\left[a(s)+\xi|u(s)|^{\rho}\right] d s \\
& +\frac{1}{|b|} \int_{t_{p}}^{T} \frac{(T-s)^{\alpha-2}}{\Gamma(\alpha-1)}\left[a(s)+\xi|u(s)|^{\rho}\right] d s+\frac{t}{T} \int_{t_{p}}^{T} \frac{(T-s)^{\alpha-1}}{\Gamma(\alpha)}\left[a(s)+\xi|u(s)|^{\rho}\right] d s \\
& +\left|\frac{\left[1+(-1)^{\theta+1}\right] t-T}{b T}\right| \sum_{i=1}^{p}\left[\int_{t_{i-1}}^{t_{i}} \frac{\left(t_{i}-s\right)^{\alpha-2}}{\Gamma(\alpha-1)}\left[a(s)+\xi|u(s)|^{\rho}\right] d s+\left|I_{i}^{*}\left(u\left(t_{i}\right)\right)\right|\right] \\
& +\left|\frac{1-(-1)^{\theta+1}-b t}{b T}\right|\left\{\sum_{i=1}^{p}\left[\int_{t_{i-1}}^{t_{i}} \frac{\left(t_{i}-s\right)^{\alpha-1}}{\Gamma(\alpha)}\left[a(s)+\xi|u(s)|^{\rho}\right] d s+\left|I_{i}\left(u\left(t_{i}\right)\right)\right|\right]\right. \\
& +\sum_{i=1}^{p-1}\left(t_{p}-t_{i}\right)\left[\int_{t_{i-1}}^{t_{i}} \frac{\left(t_{i}-s\right)^{\alpha-2}}{\Gamma(\alpha-1)}\left[a(s)+\xi|u(s)|^{\rho}\right] d s+\left|I_{i}^{*}\left(u\left(t_{i}\right)\right)\right|\right] \\
& \left.+\sum_{i=1}^{p}\left(T-t_{p}\right)\left[\int_{t_{i-1}}^{t_{i}} \frac{\left(t_{i}-s\right)^{\alpha-2}}{\Gamma(\alpha-1)}\left[a(s)+\xi|u(s)|^{\rho}\right] d s+\left|I_{i}^{*}\left(u\left(t_{i}\right)\right)\right|\right]\right\}
\end{aligned}
$$




$$
\begin{aligned}
\leq & 2(1+p)\left(1+\frac{1}{|b| T}\right) I^{\alpha} a(T)+\left[2(2 p-1) T+\frac{5 p+1}{|b|}\right] I^{\alpha-1} a(T)+2\left(1+\frac{1}{|b| T}\right) p L_{2} \\
& +\left[2(2 p-1) T+\frac{5 p-2}{|b|}\right] L_{3}+2(1+p)\left(1+\frac{1}{|b| T}\right) \frac{T^{\alpha} \xi R^{\rho}}{\Gamma(\alpha+1)} \\
& +\left[2(2 p-1) T+\frac{5 p+1}{|b|}\right] \frac{T^{\alpha-1} \xi R^{\rho}}{\Gamma(\alpha)} .
\end{aligned}
$$

Thus,

$$
\|\mathcal{G} u\| \leq \mu+v \xi R^{\rho} \leq \frac{R}{2}+\frac{R}{2}=R,
$$

where $\mu$ and $v$ are given by (3.2). This implies $\mathcal{G}: \mathbb{B} \rightarrow \mathcal{B}$. Hence, $\mathcal{G}: \mathbb{B} \rightarrow \mathbb{B}$ is completely continuous. Therefore, by the Schauder fixed-point theorem, the operator $\mathcal{G}$ has at least one fixed point. Consequently, problem (1.1) has at least one solution in $B$.

Remark 3.2. For $\rho=1$ in $\left(\mathbf{H}_{1}\right)$, if $\nu \xi<1$, we can take $R \geq \mu /(1-v \xi)$, then the conclusion of Theorem 3.1 holds.

Theorem 3.3. Suppose that there exist a nonnegative functions $a_{1} \in L(0,1)$ and a nonnegative number $\xi_{1}$ such that $|f(t, u)| \leq a_{1}(t)+\xi_{1}|u|^{\rho}$ for $\rho>1$. Furthermore, the assumption $\left(\mathbf{H}_{2}\right)$ holds. Then problem (1.1) has at least one solution.

Proof. The proof is similar to that of Theorem 3.1, so we omit it.

Theorem 3.4. Suppose that

$$
\lim _{u \rightarrow 0} \frac{f(t, u)}{u}=0, \quad \lim _{u \rightarrow 0} \frac{I_{k}(u)}{u}=0, \quad \lim _{u \rightarrow 0} \frac{I_{k}^{*}(u)}{u}=0 .
$$

Then problem (1.1) has at least one solution.

Proof. By Theorem 3.1, we know that the operator $\mathcal{G}: P C(J, \mathbb{R}) \rightarrow P C(J, \mathbb{R})$ is completely continuous. In view of (3.11), we can find a constant $r>0$ such that $|f(t, u)| \leq \delta_{1}|u|,\left|I_{k}(u)\right| \leq$ $\delta_{2}|u|$ and $\left|I_{k}^{*}(u)\right| \leq \delta_{3}|u|$ for $0<|u|<r$, where $\delta_{i}>0(i=1,2,3)$ satisfy

$$
\begin{aligned}
& \frac{2(1+p)(|b| T+1) T^{\alpha-1} \delta_{1}}{\Gamma(\alpha+1)}+\frac{[5 p+1+2|b| T(2 p-1)] T^{\alpha-1} \delta_{1}}{\Gamma(\alpha)}+\frac{2(1+|b| T) p \delta_{2}}{T} \\
& +[5 p-2+2|b| T(2 p-1)] \delta_{3} \leq|b| .
\end{aligned}
$$


Let $\Omega=\{u \in P C(J, \mathbb{R}) \mid\|u\|<r\}$. Take $u \in P C(J, \mathbb{R})$ such that $\|u\|=r$, which means $u \in \partial \Omega$. Then, as in the proof of Theorem 3.1, we have

$$
\begin{gathered}
|\mathcal{G} u(t)| \leq \frac{1}{|b|}\left\{\frac{2(1+p)(|b| T+1) T^{\alpha-1} \delta_{1}}{\Gamma(\alpha+1)}+\frac{[5 p+1+2|b| T(2 p-1)] T^{\alpha-1} \delta_{1}}{\Gamma(\alpha)}\right. \\
\left.+\frac{2(1+|b| T) p \delta_{2}}{T}+[5 p-2+2|b| T(2 p-1)] \delta_{3}\right\}\|u\|,
\end{gathered}
$$

which, in view of (3.12), implies that $\|\mathcal{G} u\| \leq\|u\|, u \in \partial \Omega$. Therefore, by Theorem 2.2, the operator $\mathcal{G}$ has at least one fixed point. Thus we conclude that problem (1.1) has at least one solution $u \in \bar{\Omega}$.

Theorem 3.5. Assume that

$\left(\mathbf{H}_{3}\right)$ there exist positive constants $K_{i}(i=1,2,3)$ such that

$$
|f(t, u)-f(t, v)| \leq K_{1}|u-v|, \quad\left|I_{k}(u)-I_{k}(v)\right| \leq K_{2}|u-v|, \quad\left|I_{k}^{*}(u)-I_{k}^{*}(v)\right| \leq K_{3}|u-v|,
$$

for $t \in J, u, v \in \mathbb{R}$ and $k=1,2, \ldots, p$.

Then problem (1.1) has a unique solution if

$$
\begin{aligned}
\Lambda= & \frac{2(1+p)(|b| T+1) T^{\alpha-1} K_{1}}{\Gamma(\alpha+1)}+\frac{[5 p+1+2|b| T(2 p-1)] T^{\alpha-1} K_{1}}{\Gamma(\alpha)}+\frac{2(1+|b| T) p K_{2}}{T} \\
& +[5 p-2+2|b| T(2 p-1)] K_{3}<|b| .
\end{aligned}
$$

Proof. For $u, v \in P C(J, \mathbb{R})$, we have

$$
\begin{aligned}
|(\mathcal{G} u)(t)-(\mathcal{G} v)(t)| \leq & \int_{t_{k}}^{t} \frac{(t-s)^{\alpha-1}}{\Gamma(\alpha)}|f(s, u(s))-f(s, v(s))| d s \\
& +\sum_{i=1}^{k}\left[\int_{t_{i-1}}^{t_{i}} \frac{\left(t_{i}-s\right)^{\alpha-1}}{\Gamma(\alpha)}|f(s, u(s))-f(s, v(s))| d s+\left|I_{i}\left(u\left(t_{i}\right)\right)-I_{i}\left(v\left(t_{i}\right)\right)\right|\right] \\
& +\sum_{i=1}^{k-1}\left(t_{k}-t_{i}\right)\left[\int_{t_{i-1}}^{t_{i}} \frac{\left(t_{i}-s\right)^{\alpha-2}}{\Gamma(\alpha-1)}|f(s, u(s))-f(s, v(s))| d s\right. \\
& \left.+\left|I_{i}^{*}\left(u\left(t_{i}\right)\right)-I_{i}^{*}\left(v\left(t_{i}\right)\right)\right|\right]
\end{aligned}
$$




$$
\begin{aligned}
& +\sum_{i=1}^{k}\left(t-t_{k}\right)\left[\int_{t_{i-1}}^{t_{i}} \frac{\left(t_{i}-s\right)^{\alpha-2}}{\Gamma(\alpha-1)}|f(s, u(s))-f(s, v(s))| d s\right. \\
& \left.+\left|I_{i}^{*}\left(u\left(t_{i}\right)\right)-I_{i}^{*}\left(v\left(t_{i}\right)\right)\right|\right] \\
& +\frac{1-(-1)^{\theta+1}}{|b| T} \int_{t_{p}}^{T} \frac{(T-s)^{\alpha-1}}{\Gamma(\alpha)}|f(s, u(s))-f(s, v(s))| d s \\
& +\frac{\left[1+(-1)^{\theta+1}\right] t}{|b| T} \int_{t_{p}}^{T} \frac{(T-s)^{\alpha-2}}{\Gamma(\alpha-1)}|f(s, u(s))-f(s, v(s))| d s \\
& +\frac{1}{|b|} \int_{t_{p}}^{T} \frac{(T-s)^{\alpha-2}}{\Gamma(\alpha-1)}|f(s, u(s))-f(s, v(s))| d s \\
& +\frac{t}{T} \int_{t_{p}}^{T} \frac{(T-s)^{\alpha-1}}{\Gamma(\alpha)}|f(s, u(s))-f(s, v(s))| d s \\
& +\left|\frac{\left[1+(-1)^{\theta+1}\right] t-T}{b T}\right| \sum_{i=1}^{p}\left[\int_{t_{i-1}}^{t_{i}} \frac{\left(t_{i}-s\right)^{\alpha-2}}{\Gamma(\alpha-1)}|f(s, u(s))-f(s, v(s))| d s\right. \\
& \left.+\left|I_{i}^{*}\left(u\left(t_{i}\right)\right)-I_{i}^{*}\left(v\left(t_{i}\right)\right)\right|\right] \\
& +\left|\frac{1-(-1)^{\theta+1}-b t}{b T}\right| \\
& \times\left\{\sum_{i=1}^{p}\left[\int_{t_{i-1}}^{t_{i}} \frac{\left(t_{i}-s\right)^{\alpha-1}}{\Gamma(\alpha)}|f(s, u(s))-f(s, v(s))| d s+\left|I_{i}\left(u\left(t_{i}\right)\right)-I_{i}\left(v\left(t_{i}\right)\right)\right|\right]\right. \\
& +\sum_{i=1}^{p-1}\left(t_{p}-t_{i}\right)\left[\int_{t_{i-1}}^{t_{i}} \frac{\left(t_{i}-s\right)^{\alpha-2}}{\Gamma(\alpha-1)}|f(s, u(s))-f(s, v(s))| d s\right. \\
& \left.+\left|I_{i}^{*}\left(u\left(t_{i}\right)\right)-I_{i}^{*}\left(v\left(t_{i}\right)\right)\right|\right] \\
& +\sum_{i=1}^{p}\left(T-t_{p}\right)\left[\int_{t_{i-1}}^{t_{i}} \frac{\left(t_{i}-s\right)^{\alpha-2}}{\Gamma(\alpha-1)}|f(s, u(s))-f(s, v(s))| d s\right. \\
& \left.\left.+\left|I_{i}^{*}\left(u\left(t_{i}\right)\right)-I_{i}^{*}\left(v\left(t_{i}\right)\right)\right|\right]\right\}
\end{aligned}
$$




$$
\begin{aligned}
& \leq \frac{1}{|b|}\left\{\frac{2(1+p)(|b| T+1) T^{\alpha-1} K_{1}}{\Gamma(\alpha+1)}+\frac{[5 p+1+2|b| T(2 p-1)] T^{\alpha-1} K_{1}}{\Gamma(\alpha)}\right. \\
& \left.\quad+\frac{2(1+|b| T) p K_{2}}{T}+[5 p-2+2|b| T(2 p-1)] K_{3}\right\}\|u-v\| \\
& =\frac{\Lambda}{|b|}\|u-v\|,
\end{aligned}
$$

which, by (3.15), yields $\|T u-T v\|<\|u-v\|$. So, $\mathcal{G}$ is a contraction. Therefore, by the Banach contraction mapping principle, problem (1.1) has a unique solution.

Example 3.6. Consider the following fractional impulsive irregular boundary value problem

$$
\begin{gathered}
{ }^{C} D^{\alpha} u(t)=\frac{e^{3 t} \cos ^{5}\left[u(t)+e^{u(t)}\right]}{1+u^{4}(t)}+\frac{\sin (t+1)}{\sqrt{5+u^{2}(t)}}|u|^{\rho}, \quad 0<t<1, \quad t \neq \frac{1}{4}, \\
\Delta u\left(\frac{1}{4}\right)=2+3 \sin ^{2}\left[\ln \left(1+2 u^{2}\left(\frac{1}{4}\right)\right)\right], \quad \Delta u^{\prime}\left(\frac{1}{4}\right)=\frac{7+2 u^{2}(1 / 4)}{2+u^{2}(1 / 4)}, \\
u^{\prime}(0)+(-1)^{\theta} u^{\prime}(1)+b u(1)=0, \quad u(0)+(-1)^{\theta+1} u(1)=0, \quad \theta=1,2, \quad b \neq 0,
\end{gathered}
$$

where $1<\alpha \leq 2$ and $p=1$.

Observe that

$$
|f(t, u)|=\left.\left.\left|\frac{e^{3 t} \cos ^{5}\left[u(t)+e^{u(t)}\right]}{1+u^{4}(t)}+\frac{\sin (t+1)}{\sqrt{5+u^{2}(t)}}\right| u\right|^{\rho}\left|\leq e^{3 t}+\right| u\right|^{\rho}
$$

Clearly, $a(t)=e^{3 t}, \xi=1, L_{2}=5, L_{3}=7 / 2$, and the conditions of Theorem 3.1 hold for $0<\rho<1$. Thus, by Theorem 3.1, problem (3.17) has at least one solution. In a similar way, for $\rho>1$, the impulsive irregular fractional boundary value problem (3.17) has at least one solution by means of Theorem 3.3.

Example 3.7. Consider the impulsive fractional irregular boundary value problem given by

$$
\begin{gathered}
{ }^{C} D^{\alpha} u(t)=t^{2}(1-\cos u(t))+e^{(3+t)} u^{4}(t), \quad 0<t<1, \quad t \neq \frac{1}{5}, \\
\Delta u\left(\frac{1}{5}\right)=\frac{\arctan ^{2} u(1 / 5)}{5}, \quad \Delta u^{\prime}\left(\frac{1}{5}\right)=e^{u^{3}(1 / 5)}-1, \\
u^{\prime}(0)+(-1)^{\theta} u^{\prime}(1)+b u(1)=0, \quad u(0)+(-1)^{\theta+1} u(1)=0, \quad \theta=1,2, \quad b \neq 0,
\end{gathered}
$$

where $1<\alpha \leq 2$ and $p=1$. 
It can easily be verified that all the assumptions of Theorem 3.4 are satisfied. Thus, by the conclusion of Theorem 3.4, we deduce that the problem (3.19) has at least one solution.

Example 3.8. Consider

$$
\begin{gathered}
{ }^{C} D^{7 / 4} u(t)=100 t^{5}+\frac{t^{2}}{200} e^{-\cos ^{2} u(t)}, \quad 0<t<1, t \neq \frac{3}{4}, \\
\Delta u\left(\frac{3}{4}\right)=\frac{1}{9} \cos u\left(\frac{3}{4}\right), \quad \Delta u^{\prime}\left(\frac{3}{4}\right)=\frac{|u(3 / 4)|}{5(1+|u(3 / 4)|)}, \\
u^{\prime}(0)+(-1)^{\theta} u^{\prime}(1)+8 u(1)=0, \quad u(0)+(-1)^{\theta+1} u(1)=0, \quad \theta=1,2 .
\end{gathered}
$$

Here $q=7 / 4, b=8, T=1$, and $p=1$. With

$$
K_{1}=\frac{1}{200}, \quad K_{2}=\frac{1}{9}, \quad K_{3}=\frac{1}{5},
$$

we find that

$$
\begin{aligned}
\Lambda= & \frac{2(1+p)(|b| T+1) T^{\alpha-1} K_{1}}{\Gamma(\alpha+1)}+\frac{[5 p+1+2|b| T(2 p-1)] T^{\alpha-1} K_{1}}{\Gamma(\alpha)}+\frac{2(1+|b| T) p K_{2}}{T} \\
& +[5 p-2+2|b| T(2 p-1)] K_{3}<6.031587<|b|=8 .
\end{aligned}
$$

Thus, all the conditions of Theorem 3.5 are satisfied. Consequently, the conclusion of Theorem 3.5 applies and the fractional order impulsive irregular boundary value problem (3.20) has a unique solution on $[0,1]$.

\section{Acknowledgment}

The research of G. Wang and L. Zhang was supported by the Natural Science Foundation for Young Scientists of Shanxi Province (2012021002-3), China.

\section{References}

[1] A. A. Kilbas, H. M. Srivastava, and J. J. Trujillo, Theory and Applications of Fractional Differential Equations, vol. 204 of North-Holland Mathematics Studies, Elsevier Science B.V., Amsterdam, The Netherlands, 2006.

[2] I. Podlubny, Fractional Differential Equations, Academic Press, San Diego, Calif, USA, 1999.

[3] J. Sabatier, O. P. Agrawal, and J. A. T. Machado, Eds., Advances in Fractional Calculus: Theoretical Developments and Applications in Physics and Engineering, Springer, Dordrecht, The Netherlands, 2007.

[4] S. G. Samko, A. A. Kilbas, and O. I. Marichev, Fractional Integrals and Derivatives, Gordon and Breach Science Publishers, Yverdon, Switzerland, 1993, Theory and Applications.

[5] R. P. Agarwal, V. Lakshmikantham, and J. J. Nieto, "On the concept of solution for fractional differential equations with uncertainty," Nonlinear Analysis. Theory, Methods E Applications A, vol. 72, no. 6, pp. 2859-2862, 2010.

[6] R. P. Agarwal, Y. Zhou, and Y. He, "Existence of fractional neutral functional differential equations," Computers E Mathematics with Applications, vol. 59, no. 3, pp. 1095-1100, 2010. 
[7] B. Ahmad and S. Sivasundaram, "On four-point nonlocal boundary value problems of nonlinear integro-differential equations of fractional order," Applied Mathematics and Computation, vol. 217, no. 2, pp. 480-487, 2010.

[8] B. Ahmad, S. K. Ntouyas, and A. Alsaedi, "New existence results for nonlinear fractional differential equations with three-point integral boundary conditions," Advances in Difference Equations, vol. 2011, Article ID 107384, 11 pages, 2011.

[9] B. Ahmad and J. J. Nieto, "Existence of solutions for nonlocal boundary value problems of higherorder nonlinear fractional differential equations," Abstract and Applied Analysis, vol. 2009, Article ID 494720, 9 pages, 2009.

[10] B. Ahmad and J. J. Nieto, "Existence results for nonlinear boundary value problems of fractional integrodifferential equations with integral boundary conditions," Boundary Value Problems, vol. 2009, Article ID 708576, 11 pages, 2009.

[11] B. Ahmad and J. J. Nieto, "Existence results for a coupled system of nonlinear fractional differential equations with three-point boundary conditions," Computers \& Mathematics with Applications, vol. 58, no. 9, pp. 1838-1843, 2009.

[12] X. Zhang, L. Liu, and Y. H. Wu, "The eigenvalue problem for a singular higher order fractional differential equation involving fractional derivatives," Applied Mathematics and Computation, vol. 218, pp. 8526-8536, 2012.

[13] X. Zhang, L. Liu, B. Wiwatanapataphee, and Y. H. Wu, "Positive solutions of eigenvalue problems for a class of fractional differential equations with derivatives," Abstract and Applied Analysis, vol. 2012, Article ID 512127, 16 pages, 2012.

[14] X. Zhang, L. Liu, and Y. H. Wu, "Multiple positive solutions of a singular fractional differential equation with negatively perturbed term," Mathematical and Computer Modelling, vol. 55, pp. 1263$1274,2012$.

[15] Y. Wang, L. Liu, and Y. Wu, "Positive solutions of a fractional boundary value problem with changing sign nonlinearity," Abstract and Applied Analysis, vol. 2012, Article ID 149849, 2012.

[16] M. Rehman and R. A. Khan, "Existence and uniqueness of solutions for multi-point boundary value problems for fractional differential equations," Applied Mathematics Letters, vol. 23, no. 9, pp. 1038$1044,2010$.

[17] M. Jia, X. Zhang, and X. Gu, "Nontrivial solutions for a higher fractional differential equation with fractional multi-point boundary conditions," Boundary Value Problems, vol. 2012, article 70, 2012.

[18] Z. Bai, "On positive solutions of a nonlocal fractional boundary value problem," Nonlinear Analysis. Theory, Methods \& Applications A, vol. 72, no. 2, pp. 916-924, 2010.

[19] D. Băleanu, O. G. Mustafa, and R. P. Agarwal, "An existence result for a superlinear fractional differential equation," Applied Mathematics Letters, vol. 23, no. 9, pp. 1129-1132, 2010.

[20] D. Băleanu and O. G. Mustafa, "On the global existence of solutions to a class of fractional differential equations," Computers \& Mathematics with Applications, vol. 59, no. 5, pp. 1835-1841, 2010.

[21] S. Zhang, "Positive solutions to singular boundary value problem for nonlinear fractional differential equation," Computers and Mathematics with Applications, vol. 59, no. 3, pp. 1300-1309, 2010.

[22] J. Wang, Y. Zhou, W. Wei, and H. Xu, "Nonlocal problems for fractional integrodifferential equations via fractional operators and optimal controls," Computers and Mathematics with Applications, vol. 62, no. 3, pp. 1427-1441, 2011.

[23] J. Wang, Y. Zhou, and W. Wei, "Impulsive fractional evolution equations and optimal controls in infinite dimensional spaces," Topological Methods in Nonlinear Analysis, vol. 38, pp. 17-43, 2011.

[24] B. Ahmad, "Existence of solutions for irregular boundary value problems of nonlinear fractional differential equations," Applied Mathematics Letters, vol. 23, no. 4, pp. 390-394, 2010.

[25] V. Lakshmikantham, D. D. BaInov, and P. S. Simeonov, Theory of Impulsive Differential Equations, vol. 6, World Scientific, Singapore, 1989.

[26] A. M. Samoĭlenko and N. A. Perestyuk, Impulsive Differential Equations, vol. 14, World Scientific, Singapore, 1995.

[27] B. Krogh and N. Lynch, Eds., Hybrid Systems: Computation and Control, vol. 1790 of Lecture Notes in Computer Science, Springer, New York, NY, USA, 2000.

[28] F. Vaandrager and J. Van Schuppen, Eds., Hybrid Systems: Computation and Control, vol. 1569 of Lecture Notes in Computer Science, Springer, New York, NY, USA, 1999.

[29] P. Egbunonu and M. Guay, "Identification of switched linear systems using subspace and integer programming techniques," Nonlinear Analysis. Hybrid Systems, vol. 1, no. 4, pp. 577-592, 2007.

[30] S. Engell, G. Frehse, and E. Schnieder, Modelling, Analysis and Design of Hybrid Systems, Lecture Notes in Control and Information Sciences, Springer, Heidelberg, Germany, 2002. 
[31] C. Tomlin, G. J. Pappas, and S. Sastry, "Conflict resolution for air traffic management: a study in multiagent hybrid systems," IEEE Transactions on Automatic Control, vol. 43, no. 4, pp. 509-521, 1998.

[32] C. Altafini, A. Speranzon, and K. H. Johansson, "Hybrid control of a truck and trailer vehicle," in Hybrid Systems: Computation and Control, C. J. Tomlin and M. R. Greenstreet, Eds., vol. 2289 of Lecture Notes in Computer Science, Springer, New York, NY, USA, 2002.

[33] A. Balluchi, L. Benvenuti, M. D. di Benedetto, C. Pinello, and A. L. Sangiovanni-Vincentelli, "Automotive engine control and hybrid systems: challenges and opportunities," Proceedings of the IEEE, vol. 88, no. 7, pp. 888-911, 2000.

[34] R. Alur, C. Courcoubetis, and D. Dill, "Model checking for real-time systems," in Proceedings of the 5th Annual IEEE Symposium on Logic in Computer Science, pp. 414-425, Philadelphia, Pa, USA, 1990.

[35] J. Lygeros, D. N. Godbole, and S. Sastry, "Verified hybrid controllers for automated vehicles," IEEE Transactions on Automatic Control, vol. 43, no. 4, pp. 522-539, 1998.

[36] P. Varaiya, "Smart cars on smart roads: problems of control," IEEE Transactions on Automatic Control, vol. 38, no. 2, pp. 195-207, 1993.

[37] D. L. Pepyne and C. G. Cassandras, "Optimal Control of hybrid systems in manufacturing," Proceedings of the IEEE, vol. 88, no. 7, pp. 1108-1122, 2000.

[38] A. Balluchi, P. Soueres, and A. Bicchi, "Hybrid feedback control for path tracking by a boundedcurvature vehicle," in Hybrid Systems: Computation and Control, M. Di Benedetto and A. L. Sangiovanni-Vincentelli, Eds., vol. 2034 of Lecture Notes in Computer Science, Springer, New York, NY, USA, 2001.

[39] S. Engell, S. Kowalewski, C. Schulz, and O. Stursberg, "Continuous-discrete interactions in chemical processing plants," Proceedings of the IEEE, vol. 88, no. 7, pp. 1050-1068, 2000.

[40] P. J. Antsaklis and A. Nerode, "Hybrid control systems: an introductory discussion to the special issue," IEEE Transactions on Automatic Control, vol. 43, pp. 457-460, 1998.

[41] R. P. Agarwal and B. Ahmad, "Existence of solutions for impulsive anti-periodic boundary value problems of fractional semilinear evolution equations," Dynamics of Continuous, Discrete E Impulsive Systems A, vol. 18, no. 4, pp. 535-544, 2011.

[42] B. Ahmad and J. J. Nieto, "Existence of solutions for impulsive anti-periodic boundary value problemsof fractional order," Taiwanese Journal of Mathematics, vol. 15, no. 3, pp. 981-993, 2011.

[43] B. Ahmad and G. Wang, "A study of an impulsive four-point nonlocal boundary value problem of nonlinear fractional differential equations," Computers $\mathcal{E}$ Mathematics with Applications, vol. 62, no. 3, pp. 1341-1349, 2011.

[44] B. Ahmad and S. Sivasundaram, "Existence results for nonlinear impulsive hybrid boundary value problems involving fractional differential equations," Nonlinear Analysis. Hybrid Systems, vol. 3, no. 3, pp. 251-258, 2009.

[45] B. Ahmad and S. Sivasundaram, "Existence of solutions for impulsive integral boundary value problems of fractional order," Nonlinear Analysis. Hybrid Systems, vol. 4, no. 1, pp. 134-141, 2010.

[46] G. M. Mophou, "Existence and uniqueness of mild solutions to impulsive fractional differential equations," Nonlinear Analysis. Theory, Methods \& Applications A, vol. 72, no. 3-4, pp. 1604-1615, 2010.

[47] Y. Tian and Z. Bai, "Existence results for the three-point impulsive boundary value problem involving fractional differential equations," Computers \& Mathematics with Applications, vol. 59, no. 8, pp. 26012609, 2010.

[48] G. Wang, B. Ahmad, and L. Zhang, "Impulsive anti-periodic boundary value problem for nonlinear differential equations of fractional order," Nonlinear Analysis, Theory, Methods and Applications, vol. 74, no. 3, pp. 792-804, 2011.

[49] G. Wang, B. Ahmad, and L. Zhang, "Some existence results for impulsive nonlinear fractional differential equations with mixed boundary conditions," Computers and Mathematics with Applications, vol. 62, no. 3, pp. 1389-1397, 2011.

[50] X. Zhang, X. Huang, and Z. Liu, "The existence and uniqueness of mild solutions for impulsive fractional equations with nonlocal conditions and infinite delay," Nonlinear Analysis: Hybrid Systems, vol. 4, no. 4, pp. 775-781, 2010.

[51] J. X. Sun, Nonlinear Functional Analysis and Its Application, Science Press, Beijing, China, 2008. 


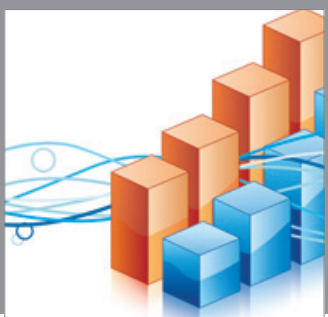

Advances in

Operations Research

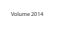

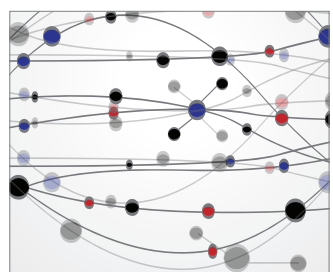

\section{The Scientific} World Journal
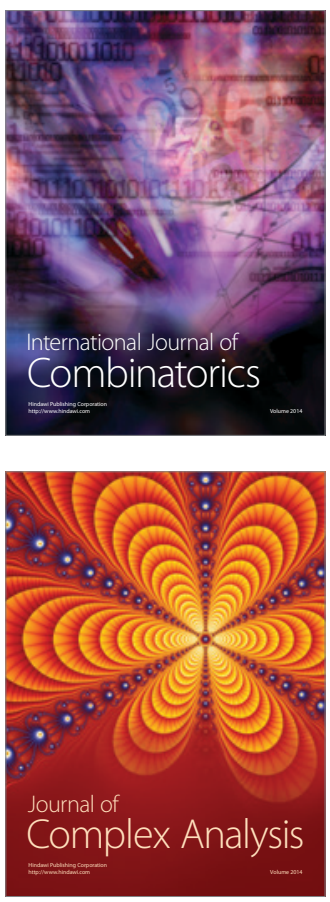

International Journal of

Mathematics and

Mathematical

Sciences
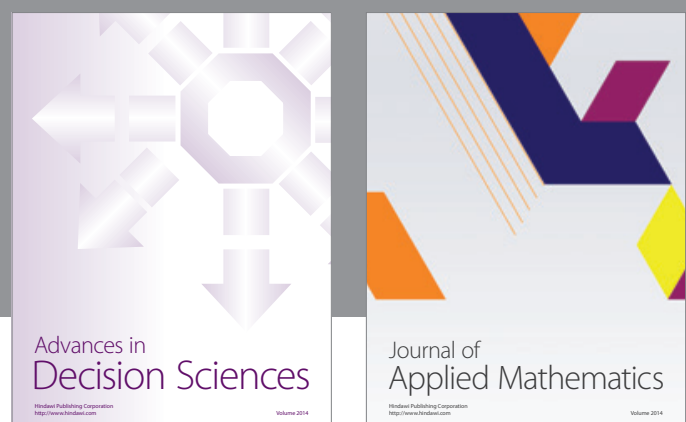

Journal of

Applied Mathematics
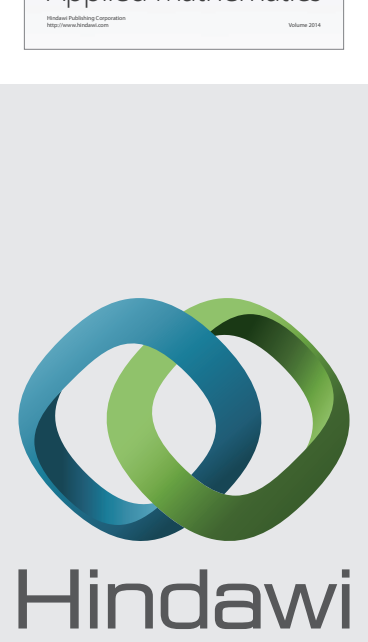

Submit your manuscripts at http://www.hindawi.com
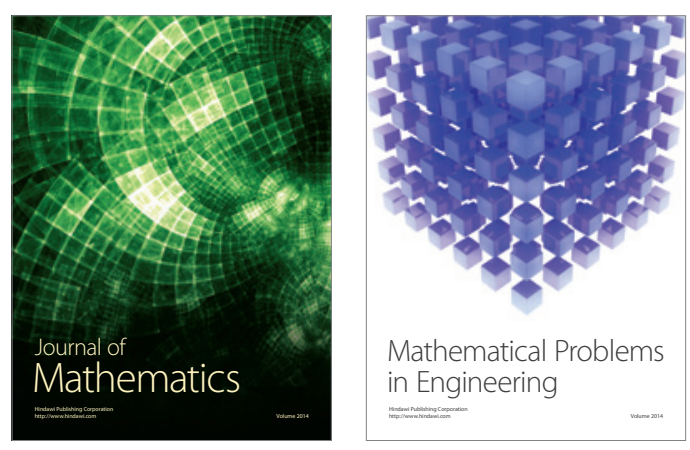

Mathematical Problems in Engineering
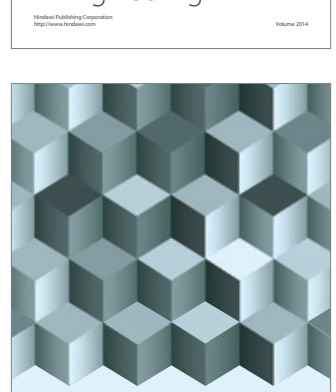

Journal of

Function Spaces
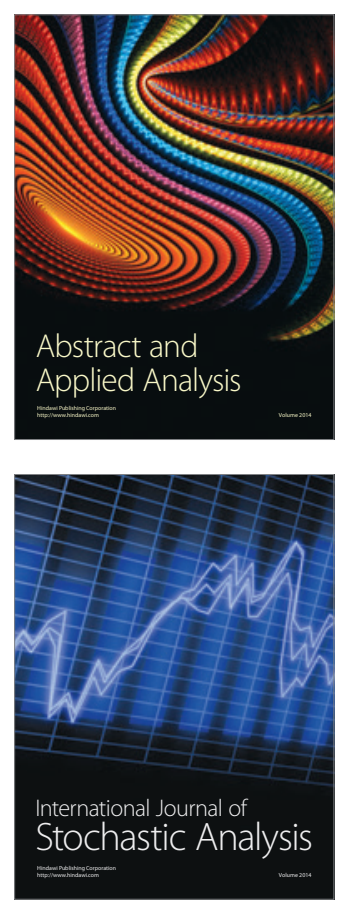

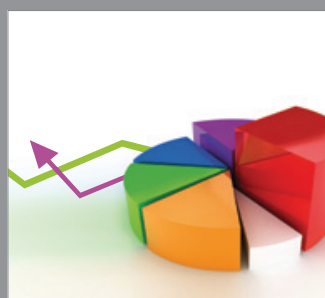

ournal of

Probability and Statistics

Promensencen
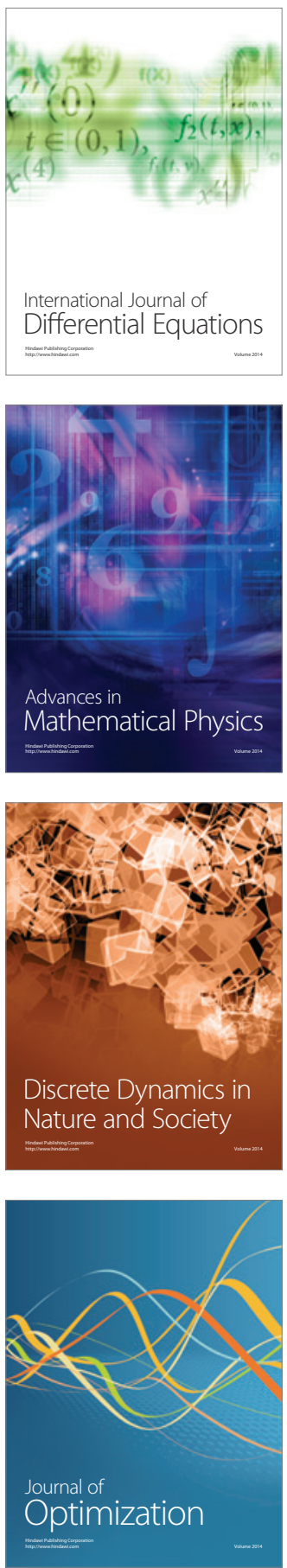